\title{
Uniform spectral radius and compact Gelfand transform
}

\author{
by \\ Alexandru Aleman and Anders Dahlner (Lund)
}

\begin{abstract}
We consider the quantization of inversion in commutative $p$-normed quasiBanach algebras with unit. The standard questions considered for such an algebra $A$ with unit $e$ and Gelfand transform $x \mapsto \widehat{x}$ are: (i) Is $K_{\nu}=\sup \left\{\left\|(e-x)^{-1}\right\|_{p}: x \in A\right.$, $\left.\|x\|_{p} \leq 1, \max |\widehat{x}| \leq \nu\right\}$ bounded, where $\nu \in(0,1)$ ? (ii) For which $\delta \in(0,1)$ is $C_{\delta}=$ $\sup \left\{\left\|x^{-1}\right\|_{p}: x \in \bar{A},\|x\|_{p} \leq 1, \min |\widehat{x}| \geq \delta\right\}$ bounded? Both questions are related to a "uniform spectral radius" of the algebra, $r_{\infty}(A)$, introduced by Björk. Question (i) has an affirmative answer if and only if $r_{\infty}(A)<1$, and this result is extended to more general nonlinear extremal problems of this type. Question (ii) is more difficult, but it can also be related to the uniform spectral radius. For algebras with compact Gelfand transform we prove that the answer is "yes" for all $\delta \in(0,1)$ if and only if $r_{\infty}(A)=0$. Finally, we specialize to semisimple Beurling type algebras $\ell_{\omega}^{p}(\mathcal{D})$, where $0<p<1$ and $\mathcal{D}=\mathbb{N}$ or $\mathcal{D}=\mathbb{Z}$. We show that the number $r_{\infty}\left(\ell_{\omega}^{p}(\mathcal{D})\right)$ can be effectively computed in terms of the underlying weight. In particular, this solves questions (i) and (ii) for many of these algebras. We also construct weights such that the corresponding Beurling algebra has a compact Gelfand transform, but the uniform spectral radius equals an arbitrary given number in $(0,1]$.
\end{abstract}

1. Introduction. This paper concerns two fundamental questions about inversion in commutative (semisimple) Banach algebras with unit which have attracted a lot of attention recently. Recall that an element $x$ in such an algebra $A$ is invertible if and only if its Gelfand transform $\widehat{x}$ has no zeros in the maximal ideal space of $A$, and it is natural to ask whether there exist quantitative versions of this fact. More precisely, we can formulate:

Question 1. Given $\delta>0$ does there exist a positive constant $C_{\delta}$ such that $\left\|x^{-1}\right\| \leq C_{\delta}$ for all $x \in A$ with $\|x\| \leq 1$ and $|\widehat{x}| \geq \delta$ ?

Question 2. Given $\varepsilon>0$ does there exist a positive constant $K_{\varepsilon}$ such that $\left\|(e-x)^{-1}\right\| \leq K_{\varepsilon}$ for all $x \in A$ with $\|x\| \leq 1$ and $|\widehat{x}| \leq \varepsilon$ ? (Here $e$ is the unit in $A$.)

2000 Mathematics Subject Classification: Primary 46J05; Secondary 43A15.

Key words and phrases: uniform spectral radius, norm controlled inversion, bounded inverse property, invisible spectrum, quasi-Banach algebras. 
We are going to consider these problems in the slightly more general context of commutative quasi-Banach algebras. The reason for this is not only because our abstract results apply to these algebras as well, but also the fact that in the concrete case of weighted $\ell^{p}$-algebras we will encounter a somewhat different situation when $0<p<1$.

A complete metric linear space $X$ over $\mathbb{C}$ is called a quasi-Banach space (also known as p-normed space or locally bounded space) if the metric is induced by a $p$-norm for some fixed $p$ with $0<p \leq 1$, i.e. a function $\|\cdot\|: X \rightarrow \mathbb{R}$ such that if $x, y \in X$ and $\lambda \in \mathbb{C}$ then: $\|x\|>0$ for $x \neq 0$, $\|\lambda x\|=|\lambda|^{p}\|x\|$, and $\|x+y\| \leq\|x\|+\|y\|$. By a quasi-Banach algebra (or $p$-normed algebra) we mean a commutative algebra $A$ over $\mathbb{C}$, with unit element $e$, such that $A$ is a quasi-Banach space with a $p$-norm satisfying $\|e\|=1$ and $\|x y\| \leq\|x\|\|y\|$ for all $x, y \in A$.

The maximal ideal space of $A$, denoted by $\mathfrak{M}=\mathfrak{M}(A)$, is the set of all (nonzero) algebra homomorphisms $\zeta: A \rightarrow \mathbb{C}$, and carries a compact Hausdorff topology [15]. We say that $A$ is semisimple if $\bigcap_{\zeta \in \mathfrak{M}} \operatorname{ker} \zeta=\{0\}$. The Gelfand transform on $A$ is the operator $\mathcal{G}: A \rightarrow C(\mathfrak{M})$ defined by $\mathcal{G}(x)=\widehat{x}$, where $\widehat{x}$ is defined by $\widehat{x}(\zeta)=\zeta(x)$, and $C(\mathfrak{M})$ is the Banach space of complex-valued continuous functions on $\mathfrak{M}$. As in the Banach algebra case, it turns out that for a complex number $\lambda, \lambda e-x \in A$ is invertible if and only if $\lambda$ does not belong to the range of the Gelfand transform of $x$. Consequently, the spectrum of an element $x \in A$, denoted by $\sigma(x)$, is the range of $\widehat{x}$, which is a compact subset of $\mathbb{C}$. The spectral radius of an element $x \in A$ is defined by $r(x)=\sup _{\zeta \in \mathfrak{M}}|\widehat{x}(\zeta)|^{p}$, or equivalently, by the spectral radius formula

$$
r(x)=\lim _{n \rightarrow \infty}\left\|x^{n}\right\|^{1 / n}=\inf _{n}\left\|x^{n}\right\|^{1 / n} .
$$

For an introduction to quasi-Banach algebras and quasi-Banach spaces we refer to Żelazko's treatise [15] and Rolewicz's book [11].

A class of examples that are relevant for this work (see Section 4 below) are the weighted $\ell^{p}$-spaces with a submultiplicative weight. They are usually called Beurling type algebras. More precisely, let $\mathcal{D}=\mathbb{N}$ or $\mathcal{D}=\mathbb{Z}$ and let $\omega$ be a strictly positive function on $\mathcal{D}$ with the property that $\omega(m+n) \leq$ $\omega(m) \omega(n), \omega(0)=1$. Such a function will be called a weight function. The space $\ell_{\omega}^{p}(\mathcal{D}), p>0$, consists of sequences $f=\left(a_{n}\right)_{n \in \mathcal{D}}$ with

$$
\|f\|_{\ell_{\omega}^{p}}=\sum_{n \in \mathcal{D}}\left|a_{n}\right|^{p} \omega(n)<\infty .
$$

The product of two such sequences is defined to be their convolution. It turns out that $\ell_{\omega}^{p}(\mathcal{D})$ is a quasi-Banach algebra if $p \leq 1$. Under certain other conditions on the weight function, $\ell_{\omega}^{p}(\mathcal{D})$ becomes a Banach algebra for $p>1$ as well, with the norm $f \mapsto\|f\|_{\ell_{\omega}^{p}}^{1 / p}$ (see [3] for more details). The 
algebras $\ell_{\omega}^{p}(\mathbb{Z})$ are always semisimple, while $\ell_{\omega}^{p}(\mathbb{N})$ is semisimple if and only if $\inf \omega(n)^{1 / n}>0$.

In his work [1] on quantization of inversion in Banach algebras Björk considered certain "uniform spectral radii" which will play a central role in this paper. These are numbers that depend only on the algebra $A$ and are defined as follows.

Definition 1.1. A sequence $\left(x_{n}\right)$ in $A$ is called a spectral null sequence provided $\left\|x_{n}\right\| \leq 1$ and $\lim _{n \rightarrow \infty} r\left(x_{n}\right)=0$. When $\boldsymbol{x}=\left(x_{n}\right)$ is a spectral null sequence and $N$ is a positive integer we define $r_{N}(\boldsymbol{x})=\varlimsup_{n \rightarrow \infty}\left\|x_{n}^{N}\right\|^{1 / N}$ and set

$$
r_{N}(A)=\sup \left\{r_{N}(\boldsymbol{x}): \boldsymbol{x} \text { is a spectral null sequence }\right\} .
$$

Moreover, we denote by $r_{\infty}(A)$ the number $\inf _{N \geq 1} r_{N}(A)=\lim _{N \rightarrow \infty} r_{N}(A)$ and refer to it as the uniform spectral radius of $A$. The existence of the limit and the last identity follow from the submultiplicativity of the sequence $\left(r_{N}(A)^{N}\right)$.

If Question 1 has an affirmative answer for some $\delta \in(0,1)$ then the quantity

$$
\begin{aligned}
C(A, \delta)=\sup \left\{\left\|x^{-1}\right\|: x \in A,\|x\| \leq\right. & 1, \\
& \left.|\widehat{x}(\zeta)|^{p} \geq \delta \text { for all } \zeta \in \mathfrak{M}(A)\right\}
\end{aligned}
$$

is finite and we say that $A$ has norm controlled inversion of degree $\delta_{1}(A)$, where $\delta_{1}(A)$ is the first critical constant

$$
\delta_{1}(A)=\inf \{\delta: \delta \in(0,1), C(A, \delta)<\infty\} .
$$

The quantity $\delta_{1}(A)$ was introduced and studied by Nikolski in [8]. It has been pointed out to us by the referee that the first explicit appearance of the problem of norm controlled inversion is the paper of J. Stafney [13] who considered the special case of the Wiener algebra $\ell^{1}(\mathbb{Z})$.

If Question 2 has an affirmative answer for some $\varepsilon \in(0,1)$ we say that $A$ has the bounded inverse property and write

$$
K_{\varepsilon}(A)=\sup \left\{\left\|(e-x)^{-1}\right\|: x \in A,\|x\| \leq 1,|\widehat{x}(\zeta)| \leq \varepsilon \text { for all } \zeta \text { in } \mathfrak{M}(A)\right\} .
$$

Both the bounded inverse property and that of norm controlled inversion have been extensively studied in Banach algebras (see [1], [2], [3], [8], [10], and [12]), that is, in the case when $p=1$. For a comprehensive survey of the problems under consideration and their relation to classical problems in the theory of harmonic analysis we refer to [9].

Due to nonlinearity such extremal problems are rather difficult and have surprising answers in many concrete cases. For example, if $A$ is one of the Wiener algebras $\ell^{1}(\mathbb{N})$ or $\ell^{1}(\mathbb{Z})$, then $K_{\varepsilon}(A)=\infty$ for all $\varepsilon>0, \delta_{1}\left(\ell^{1}(\mathbb{N})\right)=$ $1 / 2$ and $1 / 2 \leq \delta_{1}\left(\ell^{1}(\mathbb{Z})\right) \leq 1 / \sqrt{2}$, where the exact value of the last constant 
seems to be unknown (see for example [3], [9]). In fact, as we shall see below, this bad behavior of the inversion in these algebras can be explained by the fact that the uniform spectral radius $r_{\infty}(A)$ takes the maximal value 1 . A considerable amount of work has been devoted to the intrinsic relation between Björk's uniform spectral radius and the quantities described above. The aim of the present paper is to continue this investigation; we present some new results in this direction that hold even in the more general context of quasi-Banach algebras. Furthermore, the results in the last section about Beurling type algebras show that essential differences can occur when dealing with the case $p<1$.

As we mentioned above, Björk introduced the numbers $r_{N}(A)$ (for Banach algebras) and the main theorem of [1] asserts that a commutative Banach algebra $A$ has the bounded inverse property if and only if $r_{N}(A)<1$ for some integer $N \geq 2$, or equivalently $r_{\infty}(A)<1$. The argument in [1] provides an estimate of $K_{\varepsilon}(A)$ in terms of $r_{\infty}(A)$ and recently, in [10], Olofsson was able to obtain estimates of $r_{\infty}(A)$ in terms of $K_{\varepsilon}(A)$. Moreover, in the same paper Olofsson estimated from below the critical constant $\delta_{1}(A)$ in terms of the same number $r_{\infty}(A)$. Explicitly, he proved that

$$
\frac{r_{\infty}(A)}{1+r_{\infty}(A)} \leq \delta_{1}(A)
$$

In Section 2 we give a unified approach to these problems that not only works for quasi-Banach algebras but also applies to more general nonlinear extremal problems. We should point out here that the main technical difficulty when working with the case $p<1$ is that one needs a replacement for the integration techniques that are quite common in Banach algebras. The main result of the section provides two estimates of $\left\|x^{n}\right\|$ in terms of the numbers $r_{N}(A)$, as well as in terms of norms of power series applied to $x$. A consequence (see Corollary 2.3) is that for any power series $\psi$ in the unit disc whose coefficients are positive and bounded away from zero the number

$$
K(\psi, \eta)=\sup \{\|\psi(x)\|: x \in A,\|x\| \leq 1, r(x)<\eta\} \quad(\eta \in(0,1))
$$

is finite if and only if $r_{\infty}(A)<1$. Our method also yields quantitative estimates of $K(\psi, \eta)$ if $r_{\infty}(A)<1$ and of $r_{\infty}(A)$ if $K(\psi, \eta)<\infty$. A further consequence is the fact that inequality (3) continues to hold for any quasiBanach algebra.

A more subtle problem is to find estimates of $\delta_{1}(A)$ from above (see [8] and [3]) and this is due to the more complicated condition imposed on the Gelfand transform in the extremal problem that defines these numbers. An important tool for showing that $\delta_{1}(A)=0$ is the use of compact embeddings in larger algebras that have the same maximal ideal space. Such methods 
have been used by El-Fallah, Nikolski and Zarrabi [3] for certain sequence Banach algebras $A$ that contain some of the Beurling type algebras.

Note that the weakest form of compact embedding in an algebra with the same maximal ideal space is the one in $C(\mathfrak{M}(A))$ via the Gelfand transform, and also recall that by $(3)$ we have $r_{\infty}(A)=0$ whenever $\delta_{1}(A)=0$. The aim of our third section is to prove that for every semisimple quasi-Banach algebra with compact Gelfand transform this implication can be reversed, i.e. $\delta_{1}(A)=0$ whenever $r_{\infty}(A)=0$. For certain Beurling type algebras this result has been proved recently by El-Fallah and Ezzaaraoui [2].

Section 4 is devoted to algebras of semisimple Beurling type in the case $0<p<1$. We prove a general estimate of $\left\|f^{N}\right\|_{\ell_{\omega}^{p}}$ for $f \in \ell_{\omega}^{p}(\mathcal{D})$ that yields good estimates for the uniform spectral radii $r_{N}\left(\ell_{\omega}^{p}(\mathcal{D})\right)$. These estimates are then used to obtain an explicit formula for the number $r_{\infty}\left(\ell_{\omega}^{p}(\mathcal{D})\right)$ in terms of the weight $\omega$. To avoid some necessary normalizations, let us state our formula only in the case when $\mathcal{D}=\mathbb{N}$ and $\overline{\lim }_{n} \omega(n)^{1 / n}=1$. Then (see Corollary 4.3) $r_{\infty}\left(\ell_{\omega}^{p}(\mathcal{D})\right)=0$ whenever the weight is bounded, and if $\omega$ is unbounded then

$$
r_{\infty}\left(\ell_{\omega}^{p}(\mathcal{D})\right)=\inf _{k \geq 1} \varlimsup_{\omega(n) \rightarrow \infty} \frac{\omega(n k)^{1 / k}}{\omega(n)} .
$$

For many weights we have $r_{\infty}\left(\ell_{\omega}^{p}(\mathcal{D})\right)=\inf _{k \geq 1} \varlimsup_{|n| \rightarrow \infty} \omega(n k)^{1 / k} / \omega(n)=0$ and it turns out that this condition automatically implies the compactness of the Gelfand transform. Then the main result of Section 3 applies and we obtain $\delta_{1}\left(\ell_{\omega}^{p}(\mathcal{D})\right)=0$. Nevertheless, the converse to the above statement is false. For any given $\alpha \in(0,1]$ we construct a weight $\omega_{\alpha}$ such that the Gelfand transform on $\ell_{\omega_{\alpha}}^{p}(\mathcal{D})$ is compact, but $r_{\infty}\left(\ell_{\omega_{\alpha}}^{p}(\mathcal{D})\right)=\alpha$ for all $p \in(0,1]$.

These results are not only sharper than the ones known for $p \geq 1$, but they also reveal a somewhat different situation for these Beurling algebras. Indeed, for $p \geq 1$ only certain estimates in terms of more complicated expressions of the uniform spectral radius are available. Moreover, for bounded weights $\omega$ the uniform spectral radius of $\ell_{\omega}^{p}(\mathcal{D})$ can assume the maximal value 1 when $p \geq 1$.

2. General estimates of $\left\|x^{n}\right\|$. Recall that $A$ denotes a commutative semisimple quasi-Banach algebra, with unit $e$ and with a $p$-norm $\|\cdot\|$, where $p$ is some number in $(0,1]$. In this section we are going to prove two estimates of the norms of the positive powers of an element $x \in A$ with given norm and spectral radius. These inequalities will then be applied to obtain several estimates of the constants $K_{\varepsilon}(A)$ and $\delta_{1}(A)$ in terms of the uniform spectral radius $r_{\infty}(A)$ introduced in the previous section. In fact, we will consider a more general version of $K_{\varepsilon}(A)$ defined as follows. For an analytic function $\psi$ 
in the unit disc and a number $\eta \in(0,1)$ let

$$
K(\psi, \eta)=\sup \{\|\psi(x)\|: x \in A,\|x\| \leq 1, r(x)<\eta\} .
$$

Note that if $K\left(\psi, \eta_{0}\right)<\infty$ for some $\eta_{0} \in(0,1)$, then $K(\psi, \eta)<\infty$ for all $\eta$ with $0<\eta<\eta_{0}$, and the limit

$$
K_{\psi}=\lim _{\eta \rightarrow 0} K(\psi, \eta)
$$

exists.

For Banach algebras, estimates of the type mentioned above rely frequently on integration techniques which are not available in the more general context considered here. Instead, our main tool will be the following variant of the maximum principle due to Kalton [7] for $A$-valued analytic functions. As usual, for a $p$-normed space $X$ and an open subset $\Omega$ of $\mathbb{C}$, we say that a function $f: \Omega \rightarrow X$ is analytic on $\Omega$ if for each $z_{0} \in \Omega$ there exists $\delta>0$ such that for all $z$ with $\left|z-z_{0}\right|<\delta, f(z)$ can be written as a convergent power series

$$
f(z)=\sum_{n=0}^{\infty} \widehat{f}(n)\left(z-z_{0}\right)^{n}
$$

with coefficients $\widehat{f}(n) \in X$ for $n \geq 0$. The classical maximum modulus theorem is in general not true for quasi-Banach spaces (see [6] and [7] for details). However, the following weaker result holds:

THEOREM 2.1 (Theorem 5.2 of [7]). Let $X$ be a quasi-Banach space, and let $0<r<1$. Then there exists a constant $M=M(X, r)$ such that

$$
\|f(0)\| \leq M \max _{r \leq|z| \leq 1}\|f(z)\|
$$

for all analytic functions $f: \mathbb{D} \rightarrow X$ which are continuous on $\overline{\mathbb{D}}$.

We now turn to the main result of this section. We shall denote by $[a]$ the integer part of the real number $a$.

TheOREM 2.2. Let $x \in A$ with $\|x\| \leq 1$ and $r(x)=t<1$. Then:

(i) Given a positive integer $N$ and $\varepsilon>0$ there exists $0<\tau=\tau(\varepsilon, N)<1$ such that for all integers $n \geq N([\log \tau / \log t]+1)$ we have

$$
\left\|x^{n}\right\| \leq\left(r_{N}(A)+\varepsilon\right)^{k(n)},
$$

where $k(n)=k(n, N, \varepsilon)=(n+1)([\log \tau / \log t]+1)^{-1}-N$.

(ii) Given an analytic function $\psi$ in the unit disc and $\varepsilon>0$ there exists a positive constant $M_{\varepsilon}$ depending only on $\varepsilon$ such that for all positive integers $n$,

$$
|\widehat{\psi}(n)|^{p}\left\|x^{n}\right\| \leq M_{\varepsilon}(1-\varepsilon)^{-n p}(n+1)^{1-p} \sup _{1-\varepsilon<|z|<1}\|\psi(z x)\|
$$


Proof. (i) If $r_{N}(A)+\varepsilon \geq 1$ there is nothing to prove. Assume that $r_{N}(A)$ $+\varepsilon<1$ and use the definition of $r_{N}(A)$ to conclude that there exists $0<\tau$ $=\tau(\varepsilon, N)<1$ such that whenever $y \in A$ satisfies $\|y\| \leq 1, r(y)<\tau$ we have

$$
\left\|y^{N}\right\| \leq\left(r_{N}(A)+\varepsilon\right)^{N} \text {. }
$$

Now let $x$ be as in the statement and set $m=[\log \tau / \log t]+1$. Then $r\left(x^{m}\right)=$ $t^{m}<\tau$ and if $n \geq m N$ is an integer write $n=\ln N+j$ with integers $l \geq 1$ and $0 \leq j \leq m N-1$, and apply the previous inequality to $y=x^{m}$ to obtain

$$
\left\|x^{n}\right\| \leq\left\|x^{l m N}\right\| \leq\left\|x^{m N}\right\|^{l} \leq\left(r_{N}(A)+\varepsilon\right)^{l N} \leq\left(r_{N}(A)+\varepsilon\right)^{(n+1-m N) / m},
$$

proving the result.

(ii) Let $\xi=e^{2 \pi i /(n+1)}$ and consider the $A$-valued function

$$
\phi(z)=\frac{1}{(n+1) z^{n}} \sum_{k=0}^{n} \psi\left(\xi^{k} z x\right) \xi^{-k n} .
$$

We note first that this function is analytic in the punctured disc centered at the origin of radius $t^{-1}$. Moreover, if $\psi(w)=\sum_{j=0}^{\infty} \widehat{\psi}(j) w^{j}$ for $|w|<1$, then for $0<|z|<1$,

$\phi(z)=\frac{1}{z^{n}} \sum_{j=0}^{\infty} \widehat{\psi}(j)(z x)^{j} \frac{1}{n+1} \sum_{k=0}^{n} \xi^{(j-n) k}=\sum_{l=0}^{\infty} \widehat{\psi}(n+l(n+1)) z^{l(n+1)} x^{n+l(n+1)}$,

which shows that $\phi$ extends analytically near the origin as well and satisfies $\phi(0)=\widehat{\psi}(n) x^{n}$. Consequently, we may apply Theorem 2.1 to deduce that for every $\varepsilon>0$ there exists a constant $M_{\varepsilon}>0$ such that

$$
\|\phi(0)\|=|\widehat{\psi}(n)|^{p}\left\|x^{n}\right\| \leq M_{\varepsilon} \sup _{1-\varepsilon<|z|<1}\|\phi(z)\| .
$$

Now for $|z|>0$ we have the following straightforward estimate:

$$
\|\phi(z)\| \leq|z|^{-n p}(n+1)^{1-p} \sup _{|\zeta|=|z|}\|\psi(\zeta x)\|
$$

which together with the previous inequality implies the desired result.

It turns out that Theorem 2.2 can be applied to study certain nonlinear extremal problems in the algebra $A$. We shall be concerned first with the constants $K(\psi, \eta)$ defined at the beginning of the section. As pointed out in the introduction, Björk [1] and Olofsson [10] have considered this extremal problem in the case when $A$ is a Banach algebra and when $\psi(z)=(1-z)^{-1}$. The corollary below extends their results to many other functions $\psi$, that is, it provides necessary and sufficient conditions in order to have $K(\psi, \eta)<\infty$ for $\eta \in(0,1)$, as well as an estimate from above for these constants whenever they are finite. 
For an analytic function $F$ in the unit disc, we denote as above by $\widehat{F}(n)$ its $n$th Taylor coefficient and by $\widetilde{F}_{p}$ the function

$$
\widetilde{F}_{p}(z)=\sum_{n=0}^{\infty}|\widehat{F}(n)|^{p} z^{n}
$$

Corollary 2.3.

(i) If $r_{\infty}(A)<1$ then for every analytic function $\psi$ in the unit disc with $\psi(0) \neq 0$ there exist an absolute constant $\alpha>0$ and a positive constant $C_{1}$ depending only on $\psi$ such that

$$
K(\psi, \eta) \leq C_{1} \widetilde{\psi}_{p}\left(\eta^{\alpha}\right)<\infty
$$

(ii) Let $\psi$ be analytic in the unit disc. Assume that there exists an integer $s>0$ such that the Taylor coefficients, $\widehat{\psi^{s}}(n)$, of $\psi^{s}$ are real, and that there exists an integer $m \geq 0$ such that $\widehat{\psi^{s}}(n) \geq 1$ for all $n \geq m$. If $K(\psi, \eta)<\infty$ for some $0<\eta<1$ then there exists a positive constant $C_{2}$ depending only on $\psi$ such that

$$
r_{\infty}(A) \leq\left(1-\left(K_{\psi^{s}}+C_{2}\right)^{-1 / p}\right)^{p}<1 .
$$

In particular, if $\psi$ satisfies the conditions in (i) and (ii), then $K_{\psi}<\infty$ if and only if $r_{\infty}(A)<1$.

Proof. (i) Fix $\eta \in(0,1)$. Suppose that $r_{\infty}(A)<1$ and let $\varepsilon, N>0$ be such that $a=r_{N}(A)+\varepsilon<1$. Let $\tau=\tau(\varepsilon, N) \in(0,1)$ be the number given in Theorem 2.2(i) and set $N_{0}=N([\log \tau / \log \eta]+1)$. Theorem 2.2(i) states that for all $x \in A$ with $\|x\| \leq 1$ and $r(x)<\eta<1$ we have

$$
\|\psi(x)\| \leq \sum_{n=0}^{N_{0}}|\widehat{\psi}(n)|^{p}+a^{-N} \sum_{n>N_{0}}|\widehat{\psi}(n)|^{p} a^{(n+1) /([\log \tau / \log \eta]+1)} .
$$

Fix $\eta_{0}>0$ and let $\alpha>0$ satisfy

$$
\alpha \log \eta \geq \max \left\{\log a /\left(\left[\frac{\log \tau}{\log \eta}\right]+1\right),-1 / N_{0}\right\}
$$

for all $\eta \geq \eta_{0}$. For such values of $\eta$ we have

$$
\|\psi(x)\| \leq \sum_{n=0}^{N_{0}}|\widehat{\psi}(n)|^{p}+a^{-N} \sum_{n>N_{0}}|\widehat{\psi}(n)|^{p} \eta^{(n+1) \alpha}
$$

Now use the obvious inequality

$$
\sum_{n=0}^{N_{0}}|\widehat{\psi}(n)|^{p} \leq e \sum_{n=0}^{N_{0}}|\widehat{\psi}(n)|^{p} e^{-n / N_{0}} \leq e \widetilde{\psi}_{p}\left(e^{-1 / N_{0}}\right) \leq e \widetilde{\psi}_{p}\left(\eta^{\alpha}\right)
$$

to conclude that the estimate in the statement holds for all $\eta \geq \eta_{0}$ with $C_{1}=e+a^{-N}$. Finally, from the fact that $\psi(0) \neq 0$ we see that the inequality 
holds for all $\eta \in(0,1)$ with a suitable change of the constant $C_{1}$ that may now depend on $\psi$.

(ii) Suppose first that $s=1$ and $m=0$, and note that in this case we have, for all positive integers $k, n$,

$$
\widehat{\psi^{k}}(n)=\sum_{j_{1}+\cdots+j_{k}=n} \widehat{\psi}\left(j_{1}\right) \cdots \widehat{\psi}\left(j_{k}\right) \geq \sum_{j_{1}+\cdots+j_{k}=n} 1=\left(\begin{array}{c}
n+k-1 \\
n
\end{array}\right) .
$$

Choose a sequence $\left(x_{n}\right)$ in $A$ such that $\left\|x_{n}\right\| \leq 1, \eta_{n}=r\left(x_{n}\right) \rightarrow 0$ and $\left\|x_{n}^{n}\right\|^{1 / n} \rightarrow r_{\infty}(A)$ as $n \rightarrow \infty$. Apply Theorem 2.2(ii) with $\varepsilon>0$ arbitrary to obtain, for all positive integers $n, k$,

$$
\left.\widehat{\mid \psi^{k}}(n)\right|^{p}\left\|x_{n}^{n}\right\| \leq M_{\varepsilon}(1-\varepsilon)^{-n p}(n+1)^{1-p} \sup _{1-\varepsilon<|z|<1}\left\|\psi^{k}\left(z x_{n}\right)\right\| .
$$

Together with (5) this yields

$$
\left(\begin{array}{c}
n+k-1 \\
n
\end{array}\right)^{p}\left\|x_{n}^{n}\right\| \leq M_{\varepsilon}(1-\varepsilon)^{-n p}(n+1)^{1-p} K^{k}\left(\psi, \eta_{n}\right) .
$$

Now let $c>0$ be fixed but arbitrary, take the $n$th root on both sides of the above inequality and let $k, n \rightarrow \infty$ in such a manner that $|k-c n|<n^{-1}$. Then by Stirling's formula $\left(\begin{array}{c}n+k-1 \\ n\end{array}\right)^{1 / n} \rightarrow(1+c)^{1+c} c^{-c}$ and the inequality becomes

$$
r_{\infty}(A) \leq(1-\varepsilon)^{-p}\left(\frac{c}{1+c}\right)^{c p} K_{\psi}^{c}(1+c)^{-p} .
$$

If $K_{\psi}>1$, we let $c(1+c)^{-1}=K_{\psi}^{-1 / p}$, and since $\varepsilon$ was arbitrary we get

$$
r_{\infty}(A) \leq\left(1-K_{\psi}^{-1 / p}\right)^{p}
$$

The same inequality follows by a standard approximation if $K_{\psi}=1$. Finally, if $s>0, m \geq 0$ are arbitrary, let $C_{2}^{\prime}=m \max \left\{1+\left|\widehat{\psi^{s}}(n)\right|, n=0, \ldots, m-1\right\}$ and replace in the above argument $\psi$ by $\varphi(z)=\psi^{s}(z)+\left(C_{2}^{\prime} / m\right) \sum_{n=0}^{m-1} z^{n}$. Since $K(\varphi, \cdot) \leq K\left(\psi^{s}, \cdot\right)+C_{2}$, with $C_{2}=\left(C_{2}^{\prime}\right)^{p} m^{1-p}$, the result follows.

REMARK. It is easy to check that the condition imposed on $\psi$ in part (ii) is satisfied, for example, if $\widehat{\psi}(n) \geq c(n+1)^{-\gamma}$ for some constants $c>0$ and $\gamma<1$. To see this, use the inequality

$$
(k+1)^{-\gamma}(n+1-k)^{-\gamma} \geq 4^{\gamma}(n+2)^{-2 \gamma} \geq(n+1)^{-2 \gamma},
$$

which implies that

$$
\widehat{\psi^{2}}(n) \geq c^{2} \sum_{k=0}^{n}(k+1)^{-\gamma}(n+1-k)^{-\gamma} \geq c^{2}(n+1)^{-2 \gamma+1} .
$$

Since $-2 \gamma+1>-\gamma$, the result follows by iterating this inequality.

We close this section with the extension of Olofsson's estimate of $\delta_{1}(A)$ from below (see [10]) to the case of quasi-Banach algebras. 
COROLlary 2.4. The following inequality holds:

$$
\frac{r_{\infty}(A)}{1+r_{\infty}(A)} \leq \delta_{1}(A)
$$

Proof. Suppose that $\delta_{1}(A)<1$; otherwise there is nothing to prove. Let $\varepsilon>0,1>\delta>\delta_{1}(A)$ and $\left(x_{n}\right)$ be a sequence in $A$ such that $\left\|x_{n}\right\| \leq 1$, $\eta_{n}=r\left(x_{n}\right) \rightarrow 0$ and $\left\|x_{n}^{n}\right\|^{1 / n} \rightarrow r_{\infty}(A)$ as $n \rightarrow \infty$. Further, let $\varrho_{n}$ be the positive solution of the equation

$$
\frac{\left(1-\varrho_{n} \eta_{n}\right)^{p}}{1+\varrho_{n}^{p}}=\delta
$$

It follows easily from this equality that $\left(\varrho_{n}\right)$ converges to $(1 / \delta-1)^{1 / p}$ as $n \rightarrow \infty$. For $|z|<1$ set $y_{n}(z)=\left(e-z \varrho_{n} x_{n}\right)\left(1+\varrho_{n}^{p}\right)^{-1 / p}$, and note that $\left\|y_{n}(z)\right\| \leq 1$ and

$$
\left|\widehat{y_{n}(z)}\right|^{p} \geq \frac{\left(1-\varrho_{n} \eta_{n}\right)^{p}}{1+\varrho_{n}^{p}}=\delta .
$$

Then $\left\|\left(y_{n}(z)\right)^{-1}\right\| \leq C(A, \delta)$, and hence

$$
\left\|\left(e-z \varrho_{n} x_{n}\right)^{-1}\right\| \leq\left\|\left(y_{n}(z)\right)^{-1}\right\| \leq C(A, \delta) .
$$

Now apply Theorem 2.2(ii) with $\psi(z)=\left(e-z \varrho_{n} x_{n}\right)^{-1}$ to obtain the inequality

$$
\varrho_{n}^{n p}\left\|x_{n}^{n}\right\| \leq M_{\varepsilon}(1-\varepsilon)^{-n}(n+1)^{1-p} C(A, \delta) .
$$

Take the $n$th root on both sides and let $n \rightarrow \infty$ to obtain

$$
(1 / \delta-1) r_{\infty}(A) \leq(1-\varepsilon)^{-1} \text {. }
$$

Since $\varepsilon>0$ and $1>\delta>\delta_{1}(A)$ were arbitrary, the result follows.

In view of Theorem 2.2 one might be tempted to consider in the above proof other analytic functions than $z \mapsto(e-z \varrho x)^{-1}$ and try to refine the inequality. It seems, however, that this particular choice is optimal, at least for our method of proof. Furthermore, there are even Banach algebras $A$ such that $\delta_{1}(A)=1$ (see [4] and [8]) and hence the inequality in Corollary 2.4 can be strict.

3. Algebras with compact Gelfand transform. As we have seen in the previous section (Corollary 2.4), in order to have $\delta_{1}(A)=0$ for a quasi-Banach algebra $A$ it is necessary that $r_{\infty}(A)=0$. It is the aim of this section to show that the implication can be reversed for any algebra $A$ with a compact Gelfand transform. More precisely, we shall prove the following result.

THEOREM 3.1. Let $A$ be a semisimple quasi-Banach algebra such that the Gelfand transform $\mathcal{G}: A \rightarrow C(\mathfrak{M}(A))$ is compact. Then $\delta_{1}(A)=0$ if and only if $r_{\infty}(A)=0$. 
The proof is essentially based on two intermediate steps. The first is contained in the following lemma.

LEMma 3.2. Let $A$ be a semisimple quasi-Banach algebra such that $r_{\infty}(A)=0$. Further, let $x \in A$ and $\left(x_{n}\right)$ be a bounded sequence of invertible elements of $A$ such that the sequence $\left(\widehat{x}_{n}\right)$ converges to $\widehat{x}$ uniformly on $\mathfrak{M}(A)$. If $x$ is invertible then $\sup _{n}\left\|x_{n}^{-1}\right\|<\infty$.

Proof. Start with the identity

$$
x_{n}^{-1}=\sum_{j=0}^{m-1}\left(x-x_{n}\right)^{j} x^{-j-1}+x_{n}^{-1}\left(x-x_{n}\right)^{m} x^{-m}
$$

and note that it suffices to prove that there exists a positive integer $m$ such that

$$
\gamma_{m}=\varlimsup_{n \rightarrow \infty}\left\|\left(x-x_{n}\right)^{m} x^{-m}\right\|<1
$$

Indeed, assume that (7) holds for some integer $m$, let $0<\varepsilon<1-\gamma_{m}$ and use (6) to obtain, for sufficiently large $n$,

$$
\left\|x_{n}^{-1}\right\| \leq \sum_{j=0}^{m-1}\left\|\left(x-x_{n}\right)^{j} x^{-j-1}\right\|+\left(\gamma_{m}+\varepsilon\right)\left\|x_{n}^{-1}\right\| .
$$

If we define $C=\sup _{n}\left\|x_{n}\right\|+1$ this leads to

$$
\left(1-\gamma_{m}-\varepsilon\right)\left\|x_{n}^{-1}\right\| \leq \sum_{j=0}^{m-1}(\|x\|+C)^{j}\left\|x^{-1}\right\|^{j+1},
$$

and the result follows. To prove (7) note that the sequence $\left(y_{n}\right)$ defined by

$$
y_{n}=(\|x\|+C)^{-1 / p}\left\|x^{-1}\right\|^{-1 / p}\left(x-x_{n}\right) x^{-1}
$$

is a spectral null sequence in $A$. Since $r_{\infty}(A)=0$ we can choose $m$ such that

$$
r_{m}(A) \leq \frac{1}{(\|x\|+C)^{2}\left\|x^{-1}\right\|},
$$

and thus

$$
\varlimsup_{n \rightarrow \infty}\left\|y_{n}^{m}\right\|^{1 / m} \leq \frac{1}{(\|x\|+C)^{2}\left\|x^{-1}\right\|},
$$

which implies

$$
\gamma_{m}=\varlimsup_{n \rightarrow \infty}\left\|\left(x-x_{n}\right)^{m} x^{-m}\right\| \leq \frac{1}{(\|x\|+C)^{m}},
$$

and the proof is complete.

Of course, the compactness of the Gelfand transform, as assumed in Theorem 3.1, does not necessarily lead to the situation considered in the lemma. For this reason, our next aim is to enlarge our algebra $A$ in a suitable way. 
Let $\widetilde{A}$ be the set of all $x \in C(\mathfrak{M}(A))$ such that there is a bounded sequence $\left(x_{n}\right)$ in $A$ whose Gelfand transform $\left(\widehat{x}_{n}\right)$ converges to $x$ uniformly on $\mathfrak{M}(A)$. On $\widetilde{A}$ we define the $p$-norm

$$
\|x\|_{\widetilde{A}}=\inf \underset{n \rightarrow \infty}{\lim _{n \rightarrow \infty}}\left\|x_{n}\right\|_{A},
$$

where the infimum is taken over all bounded sequences $\left(x_{n}\right)$ as above.

This construction is quite common in the theory of Banach algebras and is usually called relative completion (see for example [14]). More precisely, $\widetilde{A}$ is the relative completion of $A$ with respect to $C(\mathfrak{M}(A))$. Clearly, $A$ can be identified with a subalgebra of $\widetilde{A}$ via the Gelfand transform and this will be done in what follows, in order to simplify the notation. Sometimes $\widetilde{A}$ coincides with $A$ via the above identification. A simple example where the two algebras are different is obtained for $A=\operatorname{lip}_{\alpha}(\mathbb{T})$, in which case $\widetilde{A}=\operatorname{Lip}_{\alpha}(\mathbb{T})$. The construction has been extensively used in harmonic analysis. More precisely, the so-called tilde-algebras are obtained exactly by the above procedure, starting with quotient algebras on compact groups (see [5, Chapter 12]). In particular, it turns out that $A$ may not even be closed in $\widetilde{A}$.

Let us collect some properties of the algebra $\widetilde{A}$.

Proposition 3.3. If $A$ is a semisimple quasi-Banach algebra then:

(i) $\widetilde{A}$ is a quasi-Banach algebra.

(ii) $\mathfrak{M}(\widetilde{A})=\mathfrak{M}(A)$.

(iii) $r_{N}(\widetilde{A}) \leq r_{N}(A)$.

Proof. (i) This fact is known (see [14, p. 94]); for the convenience of the reader we include a sketch of the argument. Note first that if $\left(x_{n}\right)$ is a Cauchy sequence in $\widetilde{A}$ then it must converge uniformly on $\mathfrak{M}(A)$ to some $x \in \widetilde{A}$. It also follows immediately from the definition that $\|x\|_{\widetilde{A}} \leq \underline{\lim }\left\|x_{n}\right\|_{\widetilde{A}}$. If we now fix a positive integer $k$ and apply this last inequality to the sequence $\left(x_{n}-x_{k}\right)$ we see that

$$
\varlimsup_{k \rightarrow \infty}\left\|x-x_{k}\right\|_{\widetilde{A}} \leq \varlimsup_{k \rightarrow \infty} \varliminf_{n \rightarrow \infty}\left\|x_{n}-x_{k}\right\|_{\widetilde{A}}=0 .
$$

(ii) The proof is straightforward.

(iii) Choose a spectral null sequence $\left(x_{n}\right)$ in $\widetilde{A}$ with

$$
r_{N}(\widetilde{A}) \leq \varlimsup_{n \rightarrow \infty}\left\|x_{n}^{N}\right\|_{\widetilde{A}}^{1 / N}+\varepsilon, \quad \varepsilon>0 .
$$

By the definition of $\widetilde{A}$ we can find a sequence $\left(y_{n}\right)$ in $A$ such that $\left|\widehat{x}_{n}-\widehat{y}_{n}\right|$ $<1 / n$ on $\mathfrak{M}(A)$ and

$$
\left\|y_{n}\right\| \leq 1+1 / n, \quad\left\|x_{n}^{N}\right\|_{\widetilde{A}}^{1 / N} \leq\left\|y_{n}^{N}\right\|^{1 / N}+1 / n .
$$


Clearly, $\left((1+1 / n)^{-p} y_{n}\right)$ is a spectral null sequence in $A$, and thus

$$
r_{N}(\widetilde{A}) \leq \varlimsup_{n \rightarrow \infty}\left\|x_{n}^{N}\right\|_{\widetilde{A}}^{1 / N}+\varepsilon \leq \varlimsup_{n \rightarrow \infty}\left\|y_{n}^{N}\right\|^{1 / N}+\varepsilon \leq r_{N}(A)+\varepsilon,
$$

which concludes the proof.

An immediate consequence of (i) and (ii) is that every invertible element of $A$ is invertible in $\widetilde{A}$.

Proof of Theorem 3.1. We have to show that given $\delta>0$ and a sequence $\left(x_{n}\right)$ in $A$ with $\left\|x_{n}\right\| \leq 1$ and $\left|\widehat{x}_{n}\right|^{p} \geq \delta$ we have $\sup _{n}\left\|x_{n}^{-1}\right\|<\infty$. Since the Gelfand transform is compact we can assume, by passing to a subsequence if necessary, that $\left(\widehat{x}_{n}\right)$ converges uniformly on $\mathfrak{M}(A)$ to some $x \in \widetilde{A}$. Note that if $x \in A$ the statement follows by a direct application of Lemma 3.2. If this is not the case, we can still apply Lemma 3.2 to conclude that $C_{1}=$ $\sup _{n}\left\|x_{n}^{-1}\right\|_{\widetilde{A}}<\infty$. By the definition of $\widetilde{A}$ this means that there exists a sequence $\left(y_{n}\right)$ in $A$ with $\left\|y_{n}\right\| \leq 2 C_{1}$ and $\left|\widehat{x}_{n}^{-1}-\widehat{y}_{n}\right|<1 / n$. In particular, the sequence $\left(z_{n}\right)$ defined by $z_{n}=x_{n} y_{n} \in A$ satisfies $\left\|z_{n}\right\| \leq 2 C_{1}$ and $\left(\widehat{z}_{n}\right)$ converges uniformly to the constant 1 on $\mathfrak{M}(A)$. Then, clearly, $z_{n}$ is invertible in $A$ for sufficiently large $n$ and another application of Lemma 3.2 gives

$$
C_{2}=\varlimsup_{n \rightarrow \infty}\left\|z_{n}^{-1}\right\|<\infty .
$$

On the other hand, we have the inequality

$$
\left\|x_{n}^{-1}\right\|=\left\|y_{n} z_{n}^{-1}\right\| \leq\left\|y_{n}\right\|\left\|z_{n}^{-1}\right\|,
$$

which implies that

$$
\varlimsup_{n \rightarrow \infty}\left\|x_{n}^{-1}\right\| \leq 2 C_{1} C_{2}
$$

and the result follows.

4. Beurling type quasi-Banach algebras. As in the introduction, we write $\mathcal{D}=\mathbb{N}$ or $\mathcal{D}=\mathbb{Z}$ and we let $\omega: \mathcal{D} \rightarrow(0, \infty)$ be a submultiplicative function with $\omega(0)=1$. We shall consider for $0<p \leq 1$ the quasi-Banach algebra $\ell_{\omega}^{p}(\mathcal{D})$ of all complex sequences $f=\left(a_{n}\right)_{n \in \mathcal{D}}$ such that

$$
\|f\|_{\ell_{\omega}^{p}}=\sum_{n \in \mathcal{D}}\left|a_{n}\right|^{p} \omega(n)<\infty
$$

where the product of two elements $f=\left(a_{n}\right)_{n \in \mathcal{D}}, g=\left(b_{n}\right)_{n \in \mathcal{D}}$ of $\ell_{\omega}^{p}(\mathcal{D})$ is defined to be the convolution

$$
f g=\left(\sum_{k, n-k \in \mathcal{D}} a_{k} b_{n-k}\right)_{n \in \mathcal{D}} .
$$


Let us write

$$
\begin{aligned}
& r_{+}=r_{+}(\omega)=\inf _{n>0} \omega(n)^{1 / n}=\lim _{n \rightarrow+\infty} \omega(n)^{1 / n}, \\
& r_{-}=r_{-}(\omega)= \begin{cases}\sup _{n>0} \omega(-n)^{-1 / n}=\lim _{n \rightarrow-\infty} \omega(n)^{1 / n} & \text { if } \mathcal{D}=\mathbb{Z}, \\
0 & \text { if } \mathcal{D}=\mathbb{N} .\end{cases}
\end{aligned}
$$

In the case $\mathcal{D}=\mathbb{N}$ we shall assume, in addition, that $r_{+}(\omega)>0$ (the example $\omega(n)=1 / n$ ! shows that $r_{+}=0$ is possible) to ensure that $\ell_{\omega}^{p}(\mathcal{D})$ is semisimple. In the case $\mathcal{D}=\mathbb{Z}$ one has $0<r_{-} \leq r_{+}<\infty$ and $\ell_{\omega}^{p}(\mathcal{D})$ is always semisimple. The maximal ideal space of $\ell_{\omega}^{p}(\overline{\mathcal{D}})$ is then identified with point evaluations, i.e. the Gelfand transform is given by

$$
\ell_{\omega}^{p}(\mathcal{D}) \ni f \mapsto \widehat{f}(\zeta)=\sum_{n \in \mathcal{D}} a_{n} \zeta^{n}
$$

where $\zeta \in \Omega(\omega)=\left\{\zeta \in \mathbb{C}: r_{-} \leq|\zeta|^{p} \leq r_{+}\right\}$. We will frequently identify an element $f \in \ell_{\omega}^{p}(\mathcal{D})$ with its Gelfand transform.

In order to simplify the exposition we introduce the function

$$
\sigma(n)= \begin{cases}\omega(n) / r_{+}^{n} & \text { if } n \geq 0 \\ \omega(n) / r_{-}^{n} & \text { if } \mathcal{D}=\mathbb{Z} \text { and } n<0\end{cases}
$$

With this notation we have for $f=\left(a_{n}\right)_{n \in \mathcal{D}} \in \ell_{\omega}^{p}(\mathcal{D})$ the coefficient estimate

$$
\left|a_{n}\right|^{p} \omega(n) \leq \sup _{\zeta \in \Omega(\omega)}|f(\zeta)|^{p} \sigma(n)
$$

Indeed, this follows from

$$
\left|a_{j}\right| r_{ \pm}^{j / p}=\frac{1}{2 \pi}\left|\int_{0}^{2 \pi} \widehat{f}\left(r_{ \pm}^{1 / p} e^{i t}\right) e^{-i j t} d t\right| \leq \frac{1}{2 \pi} \int_{0}^{2 \pi}\left|\widehat{f}\left(r_{ \pm}^{1 / p} e^{i t}\right)\right| d t \leq \sup _{\Omega(\omega)}|\widehat{f}(\zeta)|
$$

The aim of the present section is to give estimates for the uniform spectral radii $r_{N}\left(\ell_{\omega}^{p}(\mathcal{D})\right)$ in the case when $0<p<1$. These results enable us to effectively compute $r_{\infty}\left(\ell_{\omega}^{p}(\mathcal{D})\right)$ for such $p$. Using Theorem 3.1 we will apply our results to norm controlled inversion in these algebras.

The following estimate of the norms of powers of elements of $\ell_{\omega}^{p}(\mathcal{D})$ will be essential for our purposes.

THEOREM 4.1. Let $\omega: \mathcal{D} \rightarrow(0, \infty)$ be a weight function, and let $p \in$ $(0,1)$. Let $k, m, N \in \mathbb{N}$ be such that $1<k \leq m k<N$ and define

$$
F(k, m, N)=[(N-(m-1) k) /(k-1)] ! \text {. }
$$

Then for every $t>0$ and $f \in \ell_{\omega}^{p}(\mathcal{D})$ we have

$$
\begin{aligned}
\left\|f^{N}\right\|_{\ell_{\omega}^{p}} \leq & \|f\|_{\ell_{\omega}^{p}}^{N} F(k, m, N)^{p-1} \\
& +t^{k-1}\left(\begin{array}{c}
N \\
k
\end{array}\right) r(f)^{k-1}\|f\|_{\ell_{\omega}^{p}}^{N-k+1}+\sup _{\sigma(j)>t} \frac{\omega(k j)^{m}}{\omega(j)^{k m}}\|f\|_{\ell_{\omega}^{p}}^{N},
\end{aligned}
$$

where we set the supremum above to equal zero if $\{j \in \mathcal{D}: \sigma(j)>t\}=\emptyset$. 
Proof. Let us begin by introducing some notation. For a multi-index $\alpha=\left(\alpha_{1}, \ldots, \alpha_{N}\right)$ in $\mathcal{D}^{N}$ we write, as usual, $|\alpha|=\sum_{j=1}^{N} \alpha_{j}$. Given a function $u: \mathcal{D} \rightarrow \mathbb{C}$ and a multi-index $\alpha$ as above let

$$
\widetilde{u}(\alpha)=\prod_{j=1}^{N} u\left(\alpha_{j}\right) .
$$

Similarly, given a sequence $g=\left(b_{j}\right)$, for any multi-index $\alpha$ we write

$$
\widetilde{b}_{\alpha}=\prod_{j=1}^{N} b_{\alpha_{j}} .
$$

For a permutation $\chi \in S_{N}$ and a multi-index $\alpha=\left(\alpha_{1}, \ldots, \alpha_{N}\right) \in \mathcal{D}^{N}$ we write

$$
\chi \alpha=\left(\alpha_{\chi(1)}, \ldots, \alpha_{\chi(N)}\right) .
$$

For an integer $k$ with $1<k<N$ and a multi-index $\alpha$ as above we define $s(\alpha)$ as the greatest nonnegative integer $s \leq N / k$ with the property that there exists $\chi \in S_{N}$ such that $\chi \alpha$ can be written in the form

$$
\chi \alpha=(\underbrace{\beta_{1}, \ldots, \beta_{1}}_{k \text { times }}, \ldots, \underbrace{\beta_{s}, \ldots, \beta_{s}}_{k \text { times }}, \beta_{k s+1}, \ldots, \beta_{N}) .
$$

Note that for every $s \leq s(\alpha)$ and for every sequence $\left(b_{j}\right)_{j \in \mathcal{D}}$ we can write

$$
\widetilde{b}_{\alpha}=\widetilde{b}_{\beta}^{k} \widetilde{b}_{\gamma}
$$

with $\beta \in \mathcal{D}^{s}$ and $\gamma \in \mathcal{D}^{N-k s}$, where $\widetilde{b}_{\gamma}$ is taken to be 1 if $s=s(\alpha)=N / k$. Finally, observe that if $s=s(\alpha)<N / k$ then each entry of the multi-index $\gamma$, in the representation (10), occurs at most $k-1$ times, and consequently, at least $[(N-k s(\alpha)) /(k-1)]$ entries of $\gamma$ are distinct.

After these preparations we turn to the actual proof of the theorem. Start with the equality

$$
\left\|f^{N}\right\|_{\ell_{\omega}^{p}}=\sum_{j \in \mathcal{D}}\left|\sum_{\alpha \in \mathcal{D}^{N},|\alpha|=j} \widetilde{a}_{\alpha}\right|^{p} \omega(j) .
$$

Let us define the following sets of multi-indices:

1) The set $A_{j, 1}$ of multi-indices $\alpha$ with $|\alpha|=j$ and $s(\alpha)<m$.

2) The set $A_{j, 2}$ of multi-indices $\alpha$ with $|\alpha|=j, s(\alpha) \geq m$ and such that, if $\widetilde{a}_{\alpha}$ is written in the form (10) with $s=m$, then at least one of the entries $\beta_{i}$ of $\beta=\left(\beta_{1}, \ldots, \beta_{s}\right)$ satisfies $\sigma\left(\beta_{i}\right)<t$.

3) The set $A_{j, 3}$ of multi-indices $\alpha$ with $|\alpha|=j$ that do not belong to the previous sets, that is, $s(\alpha) \geq m$ and if $\widetilde{a}_{\alpha}$ is written in the form (10) with $s=m$ then all entries $\beta_{i}$ of $\beta=\left(\beta_{1}, \ldots, \beta_{s}\right)$ satisfy $\sigma\left(\beta_{i}\right) \geq t$. 
We have

$$
\left\|f^{N}\right\|_{\ell_{\omega}^{p}} \leq \sum_{\mu=1}^{3} \sum_{j \in \mathcal{D}}\left|\sum_{\alpha \in A_{j, \mu}} \widetilde{a}_{\alpha}\right|^{p} \omega(j)
$$

Recall that every multi-index in $A_{j, 1}$ has at least $[(N-(m-1) k) /(k-1)]$ distinct entries and we can write

$$
\sum_{\alpha \in A_{j, 1}} \widetilde{a}_{\alpha}=F(k, m, N) \sum_{\alpha \in A_{j, 1}}^{\prime} \widetilde{a}_{\alpha}
$$

where $\sum^{\prime}$ means that we are summing over all those multi-indices whose first $[(N-(m-1) k) /(k-1)]$ distinct entries are ordered. This implies

$$
\sum_{j \in \mathcal{D}}\left|\sum_{\alpha \in A_{j, 1}} \widetilde{a}_{\alpha}\right|^{p} \omega(j) \leq F(k, m, N)^{p} \sum_{j \in \mathcal{D}} \sum_{\alpha \in A_{j, 1}}^{\prime}\left|\widetilde{a}_{\alpha}\right|^{p} \widetilde{\omega}(\alpha) .
$$

On the other hand, the same reasoning shows that

$$
F(k, m, N) \sum_{j \in \mathcal{D}} \sum_{|\alpha|=j}^{\prime}\left|\widetilde{a}_{\alpha}\right|^{p} \widetilde{\omega}(\alpha) \leq\|f\|_{\ell_{\omega}^{p}}^{N}
$$

and we obtain the estimate

$$
\sum_{j \in \mathcal{D}}\left|\sum_{\alpha \in A_{j, 1}} \widetilde{a}_{\alpha}\right|^{p} \omega(j) \leq F(k, m, N)^{p-1}\|f\|_{\ell_{\omega}^{p}}^{N} .
$$

To handle the second sum in (12) we introduce the set $B$ of multi-indices $\alpha=\left(\alpha_{1}, \ldots, \alpha_{N}\right)$ such that $\widetilde{a}_{\alpha}$ can be written in the form

$$
\widetilde{a}_{\alpha}=a_{l}^{k} a_{l_{k+1}} \cdots a_{l_{N}},
$$

where $\sigma(l)<t$. Clearly, $\bigcup_{j \in \mathcal{D}} A_{j, 2} \subset B$, which implies the inequality

$$
\sum_{j \in \mathcal{D}}\left|\sum_{\alpha \in A_{j, 2}} \widetilde{a}_{\alpha}\right|^{p} \omega(j) \leq \sum_{\alpha \in B}\left|\widetilde{a}_{\alpha}\right|^{p} \widetilde{\omega}(\alpha)
$$

Moreover,

$$
\begin{aligned}
\sum_{\alpha \in B}\left|\widetilde{a}_{\alpha}\right|^{p} \widetilde{\omega}(\alpha) & =\left(\begin{array}{c}
N \\
k
\end{array}\right) \sum_{\sigma(l)<t}\left|a_{l}\right|^{k p} \omega(l)^{k} \sum_{\alpha \in \mathcal{D}^{N-k}}\left|\widetilde{a}_{\alpha}\right|^{p} \widetilde{\omega}(\alpha) \\
& =\|f\|_{\ell_{\omega}^{p}}^{N-k}\left(\begin{array}{c}
N \\
k
\end{array}\right) \sum_{\sigma(l)<t}\left|a_{l}\right|^{k p} \omega(l)^{k} .
\end{aligned}
$$

Now use the coefficient estimate (8) to conclude that

$$
\sum_{\sigma(l)<t}\left|a_{l}\right|^{k p} \omega(l)^{k} \leq t^{k-1} r(f)^{k-1}\|f\|_{\ell_{\omega}^{p}},
$$


which gives

$$
\sum_{j \in \mathcal{D}}\left|\sum_{\alpha \in A_{j, 2}} \widetilde{a}_{\alpha}\right|^{p} \omega(j) \leq \sum_{\alpha \in B}\left|\widetilde{a}_{\alpha}\right|^{p} \widetilde{\omega}_{\alpha} \leq\left(\begin{array}{c}
N \\
k
\end{array}\right) t^{k-1} r(f)^{k-1}\|f\|_{\ell_{\omega}^{p}}^{N-k+1} .
$$

To estimate the third sum in (12) we note that if $\alpha=\left(\alpha_{1}, \ldots, \alpha_{N}\right) \in A_{j, 3}$ and

$$
\widetilde{a}_{\alpha}=a_{l_{1}}^{k} \cdots a_{l_{m}}^{k} a_{l_{k m+1}} \cdots a_{l_{N}}
$$

then $\sigma\left(l_{\nu}\right) \geq t, 1 \leq \nu \leq m$, and hence

$$
\omega(j) \leq \widetilde{\omega}(\alpha) \prod_{\nu=1}^{m} \frac{\omega\left(k l_{\nu}\right)}{\omega\left(l_{\nu}\right)^{k}} \leq \widetilde{\omega}(\alpha) \sup _{\sigma(l)>t} \frac{\omega(k l)^{m}}{\omega(l)^{k m}} .
$$

Then

$$
\begin{aligned}
\sum_{j \in \mathcal{D}}\left|\sum_{A_{j, 3}} \widetilde{a}_{\alpha}\right|^{p} \omega(j) & \leq \sup _{\sigma(j)>t} \frac{\omega(k j)^{m}}{\omega(j)^{k m}} \sum_{j \in \mathcal{D}} \sum_{A_{j, 3}}\left|\widetilde{a}_{\alpha}\right|^{p} \widetilde{\omega}(\alpha) \\
& \leq \sup _{\sigma(j)>t} \frac{\omega(k j)^{m}}{\omega(j)^{k m}}\|f\|_{\ell_{\omega}^{p}}^{N} .
\end{aligned}
$$

The result now follows from (12) and the estimates (13)-(15).

Let us now turn to the estimates of the uniform spectral radii. To state our result we need the following notation. Given an unbounded sequence $\left(\mu_{n}\right)$ of positive numbers we define, for arbitrary sequences $\left(A_{n}\right)$,

$$
\varlimsup_{\mu_{n} \rightarrow \infty} A_{n}=\sup \left\{\varlimsup_{k \rightarrow \infty} A_{n_{k}}: \lim _{k \rightarrow \infty} \mu_{n_{k}}=\infty\right\} .
$$

We obviously have the equality

$$
\varlimsup_{\mu_{n} \rightarrow \infty} A_{n}=\lim _{t \rightarrow \infty} \sup \left\{A_{n}: \mu_{n}>t\right\} .
$$

Corollary 4.2. Let $\omega: \mathcal{D} \rightarrow(0, \infty)$ be a weight function and let $p \in(0,1)$.

(i) If $\sup _{n \in \mathcal{D}} \sigma(n)<\infty$ then for all integers $N \geq 3$ we have

$$
r_{N}\left(\ell_{\omega}^{p}(\mathcal{D})\right)^{N} \leq N !^{(p-1)} .
$$

(ii) Let $(\sigma(n))_{n \in \mathcal{D}}$ be unbounded, let $k, m, N$ be positive integers with $1<k \leq m k<N$ and set $F(k, m, N)=[(N-(m-1) k) /(k-1)]$ !. Then

$$
\varlimsup_{\sigma(n) \rightarrow \infty} \frac{\omega(n N)}{\omega(n)^{N}} \leq r_{N}\left(\ell_{\omega}^{p}(\mathcal{D})\right)^{N} \leq F(k, m, N)^{p-1}+\varlimsup_{\sigma(n) \rightarrow \infty} \frac{\omega(k n)^{m}}{\omega(n)^{k m}} .
$$

Proof. Recall that for a spectral null sequence $\boldsymbol{f}=\left(f_{n}\right)$ we have $\left\|f_{n}\right\|_{\ell_{\omega}^{p}}$ $\leq 1$ and $\lim _{n \rightarrow \infty} r\left(f_{n}\right)=0$. Then by an application of Theorem 4.1 we obtain 
the inequality

$$
r_{N}(\boldsymbol{f})^{N}=\varlimsup_{n \rightarrow \infty}\left\|f_{n}^{N}\right\|_{\ell_{\omega}^{p}} \leq F(k, m, N)^{p-1}+\sup _{\sigma(j)>t} \frac{\omega(k j)^{m}}{\omega(j)^{k m}},
$$

whenever $k, m, N, t$ satisfy the conditions in the statement. Then (i) follows by letting $k=2, m=1$ and $t>\sup _{n \in \mathcal{D}} \sigma(n)$. Recall also that in this case the supremum on the right hand side of (16) is taken to be zero.

For (ii), to see the lower estimate consider a sequence $n_{j}$ in $\mathcal{D}$ such that $\lim _{j \rightarrow \infty} \sigma\left(n_{j}\right)=\infty$ and let $f_{j}$ be defined by $\widehat{f}_{j}(z)=z^{n_{j}} / \omega\left(n_{j}\right)^{1 / p}$. Clearly $\left(f_{j}\right)$ is a spectral null sequence, and thus

$$
r_{N}\left(\ell_{\omega}^{p}(\mathcal{D})\right)^{N} \geq \varlimsup_{j \rightarrow \infty}\left\|f_{j}^{N}\right\|_{\ell_{\omega}^{p}}=\varlimsup_{j \rightarrow \infty} \frac{\omega\left(n_{j} N\right)}{\omega\left(n_{j}\right)^{N}},
$$

which gives the desired inequality. For the upper estimate we just apply (16) and let the parameter $t$ tend to infinity.

Corollary 4.3. Let $\omega: \mathcal{D} \rightarrow(0, \infty)$ be a weight function and let $p \in$ $(0,1)$. If $(\sigma(n))_{n \in \mathcal{D}}$ is bounded then $r_{\infty}\left(\ell_{\omega}^{p}(\mathcal{D})\right)=0$ and if $(\sigma(n))_{n \in \mathcal{D}}$ is unbounded then

$$
r_{\infty}\left(\ell_{\omega}^{p}(\mathcal{D})\right)=\inf _{N \geq 2} \varlimsup_{\sigma(n) \rightarrow \infty} \frac{\omega(n N)^{1 / N}}{\omega(n)} .
$$

Proof. The first part of the statement is just a direct application of Corollary 4.2(i). Further, from the first inequality in part (ii) of the same corollary we see that

$$
r_{\infty}\left(\ell_{\omega}^{p}(\mathcal{D})\right) \geq \inf _{N \geq 2} \varlimsup_{\sigma(n) \rightarrow \infty} \frac{\omega(n N)^{1 / N}}{\omega(n)} .
$$

To see the reverse inequality, fix an integer $k \geq 2$ and a constant $c \in(0,1)$, and let $m, N \rightarrow \infty$ in such a manner that $m k / N \rightarrow c$. Then

$$
\frac{N-(m-1) k}{k-1} \rightarrow \infty \text { and } \frac{N-(m-1) k}{N(k-1)} \rightarrow \frac{1-c}{k-1},
$$

which (due to Stirling's formula) implies that $F(k, m, N)^{(p-1) / N} \rightarrow 0$. Then from the second inequality in Corollary 4.2(ii) we deduce that

$$
r_{N}\left(\ell_{\omega}^{p}(\mathcal{D})\right) \leq F(k, m, N)^{(p-1) / N}+\varlimsup_{\sigma(n) \rightarrow \infty} \frac{\omega(k n)^{m / N}}{\omega(n)^{k m / N}}
$$

and by the above considerations, when $m, N \rightarrow \infty$ so that $m k / N \rightarrow c$, we have

$$
r_{\infty}\left(\ell_{\omega}^{p}(\mathcal{D})\right) \leq\left(\varlimsup_{\sigma(n) \rightarrow \infty} \frac{\omega(k n)^{1 / k}}{\omega(n)}\right)^{c} .
$$

Since $k \geq 2$ and $c \in(0,1)$ were arbitrary the result follows. 
We should point out here that the value of the infimum in Corollary 4.3 can be obtained by letting $N \rightarrow \infty$. This follows immediately from the fact that the weight is submultiplicative.

Next we want to apply Theorem 3.1 to the algebras $\ell_{\omega}^{p}(\mathcal{D}), 0<p<1$. It is fairly easy to check that the Gelfand transform of $\ell_{\omega}^{p}(\mathcal{D})$ is compact if and only if

$$
\lim _{n \in \mathcal{D},|n| \rightarrow \infty} \sigma(n)=\infty .
$$

Indeed, if the Gelfand transform is compact one can use the test functions defined by $f_{n}(z)=z^{n} \omega(n)^{-1 / p}$ to conclude that the above limit is infinite. Conversely, if the limit is infinite then the inclusion map from $\ell_{\omega}^{p}(\mathcal{D})$ into $\ell_{\omega_{0}}^{1}(\mathcal{D})$, where $\omega_{0}( \pm n)=r_{ \pm}^{n / p}, n \geq 0$, is compact. Moreover, $\ell_{\omega_{0}}^{1}(\mathcal{D})$ is continuously contained in $C(\Omega(\omega))$, which proves the compactness of the Gelfand transform. Note that for such weights $\omega$ Corollary 4.3 gives

$$
r_{\infty}\left(\ell_{\omega}^{p}(\mathcal{D})\right)=\inf _{k \geq 1} \varlimsup_{|n| \rightarrow \infty} \frac{\omega(n k)^{1 / k}}{\omega(n)} .
$$

Corollary 4.4. Let $\omega: \mathcal{D} \rightarrow(0, \infty)$ be a weight function such that

$$
\inf _{k \geq 1} \varlimsup_{|n| \rightarrow \infty} \frac{\omega(n k)^{1 / k}}{\omega(n)}=0 .
$$

Then the Gelfand transform of $\ell_{\omega}^{p}(\mathcal{D})$ is compact for $0<p \leq 1$. Moreover, if $0<p<1$, then $\delta_{1}\left(\ell_{\omega}^{p}(\mathcal{D})\right)=0$.

Proof. Let us assume $\mathcal{D}=\mathbb{N}$, the statement for $\mathcal{D}=\mathbb{Z}$ is identical. By Theorem 3.1 and Corollary 4.3 the second half of the statement follows from the first. By the remarks preceding this proof it suffices to show that $\sigma(n) \rightarrow \infty$ as $n \rightarrow \infty$. Note that the hypothesis is equivalent to

$$
\inf _{k \geq 1} \varlimsup_{n \rightarrow \infty} \frac{\sigma(n k)^{1 / k}}{\sigma(n)}=0 .
$$

Moreover, since $r_{+}=\inf _{n} \omega(n)^{1 / n}$ it follows that $\sigma(n) \geq 1$. Thus,

$$
0=\inf _{k \geq 1} \varlimsup_{n \rightarrow \infty} \frac{\sigma(n k)^{1 / k}}{\sigma(n)} \geq \varlimsup_{n \rightarrow \infty} \frac{1}{\sigma(n)}
$$

and the proof is complete.

Explicit examples of weights satisfying the assumption of Corollary 4.4 are $\omega: n \mapsto(1+|n|)^{\alpha}$ and $\omega: n \mapsto \exp \left(|n|^{\beta}\right)$, where $\alpha>0$ and $\beta \in(0,1)$. In fact, for these weights $\overline{\lim }_{n} \omega(k n) / \omega(n)^{k}=0$ for all $k>1$. Our next example shows that there are weights $\omega$ such that the Gelfand transform of $\ell_{\omega}^{p}(\mathcal{D})$ is compact, but $r_{\infty}\left(\ell_{\omega}^{p}(\mathcal{D})\right)>0$. 
EXAMPLE 4.5. Let $0<p \leq 1$. Given $\alpha \in(0,1]$ there is a weight function $\omega$ on $\mathbb{N}$ such that $\lim _{n \rightarrow \infty} \sigma(n)=\infty$ and

$$
r_{\infty}\left(\ell_{\omega}^{p}(\mathbb{N})\right)=\alpha
$$

Proof. In the case when $p \in(0,1)$ we see from Corollary 4.3 that it suffices to construct a weight function $\omega_{\alpha}: \mathbb{N} \rightarrow \mathbb{R}$ with the properties $\lim \omega_{\alpha}(n)=\infty, r_{+}(\omega)=1$ and

$$
\inf _{k \geq 1} \varlimsup_{n \rightarrow \infty} \frac{\omega_{\alpha}(n k)^{1 / k}}{\omega_{\alpha}(n)}=\alpha .
$$

Assume first that $\alpha=1$ and consider a rapidly increasing sequence of positive integers $n_{1}, n_{2}, \ldots$, where by rapidly increasing we mean that for every $k>1$ there are infinitely many $n$ such that $n_{j}<n<k n \leq n_{j+1}$ for some $j$ (depending on $n$ ). Define $f: \mathbb{N} \rightarrow(0,1)$ by $f(n)=1 / j$ if $n_{j}<n \leq n_{j+1}$ and note that $f$ has the following properties: $f(n)$ decreases to 0 as $n$ grows to infinity, $\lim n f(n)=\infty$, and for $k$ fixed we have $f(n k)-f(n)=0$ for infinitely many $n$. In other words, $\omega_{1}: n \mapsto \exp (n f(n))$ satisfies all the conditions above. To extend the result to the case when $p=1$ we use the following inequalities. If $\omega$ is a weight function on $\mathbb{N}$ with $\sigma(n) \rightarrow \infty$ then

$$
\begin{aligned}
\inf _{k \geq 1} \varlimsup_{n \rightarrow \infty} \frac{\omega(n k)^{1 / k}}{\omega(n)} & \leq r_{\infty}\left(\ell_{\omega}^{1}(\mathbb{N})\right) \\
& \leq \inf _{k \geq 1} \lim _{M \rightarrow \infty} \sup _{n_{1}, \ldots, n_{k} \geq M}\left(\frac{\omega\left(n_{1}+\cdots+n_{k}\right)}{\omega\left(n_{1}\right) \cdots \omega\left(n_{k}\right)}\right)^{1 / k} .
\end{aligned}
$$

The upper estimate follows from [2], while the lower estimate is obtained exactly as in Corollary 4.2 , using spectral null sequences $\left(f_{j}\right)$ with $\widehat{f}_{j}(z)=$ $z^{n_{j}} / \omega\left(n_{j}\right)^{1 / p}$. This completes our construction in the case when $\alpha=1$.

To see the general case, let $\alpha \in(0,1)$ and define $\omega_{\alpha}$ by $\omega_{\alpha}(0)=1$ and $\omega_{\alpha}(n)=\alpha^{-1} \omega_{1}(n)$ otherwise. Then $\lim \omega_{\alpha}(n)=\infty, r_{+}\left(\omega_{\alpha}\right)=1$ and a simple calculation based on the above estimates of $r_{\infty}\left(\ell_{\omega}^{p}(\mathbb{N})\right)$ shows that $r_{\infty}\left(\ell_{\omega_{\alpha}}^{p}(\mathbb{N})\right)=\alpha$.

The simple construction given in this example has been suggested to us by S. Naboko. It is interesting to observe that for the weight $\omega_{1}$ constructed above we have $\delta_{1}\left(\ell_{\omega}^{p}(\mathbb{N})\right)=1 / 2$. Indeed, since $r_{\infty}\left(\ell_{\omega}^{p}(\mathbb{N})\right)=1$, Corollary 2.4 implies that $\delta_{1}\left(\ell_{\omega}^{p}(\mathbb{N})\right) \geq 1 / 2$. The reverse inequality is a classical result. Its proof can be found in [3] or [8].

Proposition 4.6. If $\omega$ is a weight function on $\mathbb{N}$ and $0<p \leq 1$, then

$$
\delta_{1}\left(\ell_{\omega}^{p}(\mathbb{N})\right) \leq 1 / 2
$$


Proof. Fix $\delta>1 / 2$. Suppose $f \in \ell_{\omega}^{p}(\mathbb{N})$ satisfies $\|f\|_{\ell_{\omega}^{p}} \leq 1$ and $|\widehat{f}(z)|^{p} \geq \delta$ for all $z \in \Omega(\omega)$. Then $|\widehat{f}(0)|^{p} \geq \delta$ and

$$
\begin{aligned}
\left\|f^{-1}\right\|_{\ell_{\omega}^{p}} & =\frac{1}{|\widehat{f}(0)|^{p}}\left\|(1+(f-\widehat{f}(0)) / \widehat{f}(0))^{-1}\right\|_{\ell_{\omega}^{p}} \\
& \leq \frac{1}{|\widehat{f}(0)|^{p}} \sum_{n=0}^{\infty} \frac{\|f-\widehat{f}(0)\|_{\ell_{\omega}^{p}}^{n}}{|\widehat{f}(0)|^{n p}} \leq \frac{1}{2 \delta-1} .
\end{aligned}
$$

Acknowledgements. We are greatly indebted to the referees for suggestions and comments that have considerably improved the exposition. Also, we would like thank A. Atzmon, O. El-Fallah, S. Naboko, N. K. Nikolski, A. Olofsson, H. S. Shapiro and S. M. Shimorin for many valuable discussions and suggestions.

\section{References}

[1] J.-E. Björk, On the spectral radius formula in Banach algebras, Pacific J. Math. 40 (1972), 279-284.

[2] O. El-Fallah et A. Ezzaaraoui, Majorations uniformes de normes d'inverses dans les algèbres de Beurling, J. London Math. Soc. (2) 65 (2002), 705-719.

[3] O. El-Fallah, N. K. Nikolski and M. Zarrabi, Estimates for resolvents in BeurlingSobolev algebras, Algebra i Analiz 10 (1998), no. 6, 1-92 (in Russian); English transl.: St. Petersburg Math. J. 10 (1999), no. 6, 901-964.

[4] O. El-Fallah et M. Zarrabi, Estimations des solutions de l'équation de Bézout dans les algèbres de Beurling analytiques, Math. Scand. 96 (2005), 307-319.

[5] C. C. Graham and O. C. McGehee, Essays in Commutative Harmonic Analysis, Springer, New York, 1979.

[6] N. J. Kalton, Analytic functions in nonlocally convex spaces and applications, Studia Math. 83 (1986), 275-303.

[7] —, Plurisubharmonic functions on quasi-Banach spaces, ibid. 84 (1986), 297-324.

[8] N. K. Nikolski, In search of the invisible spectrum, Ann. Inst. Fourier (Grenoble) 49 (1999), 1925-1998.

[9] -, The problem of efficient inversions and Bézout equations, in: Twentieth Century Harmonic Analysis-A Celebration (Il Ciocco, 2000), NATO Sci. Ser. II Math. Phys. Chem. 33, Kluwer, Dordrecht, 2001, 235-269.

[10] A. Olofsson, An extremal problem in Banach algebras, Studia Math. 145 (2001), $255-264$.

[11] S. Rolewicz, Metric Linear Spaces, 2nd ed., PWN-Polish Sci. Publ., Warszawa, and Reidel, Dordrecht, 1985.

[12] H. S. Shapiro, A counterexample in harmonic analysis, in: Approximation Theory, Banach Center Publ. 4, PWN-Polish Sci. Publ., Warszawa, 1979, 233-236.

[13] J. D. Stafney, An unbounded inverse property in the algebra of absolutely convergent Fourier series, Proc. Amer. Math. Soc. 18 (1967), 497-498.

[14] H. C. Wang, Homogeneous Banach Algebras, Lecture Notes in Pure and Appl. Math. 29, Marcel Dekker, New York, 1977. 
[15] W. Żelazko, Metric generalizations of Banach algebras, Rozprawy Mat. 47 (1965).

Centre for Mathematical Sciences

Mathematics (Faculty of Science)

University of Lund, Box 118

SE-221 00 Lund, Sweden

E-mail: Alexandru.Aleman@math.lu.se

Anders.Dahlner@math.lu.se

Received April 15, 2004

Revised version August 11, 2005 